\title{
A current perspective on the relevance of nano and microplastics in the neurodevelopmental disorders: further relevance for metabolic, gastrointestinal, oxidative stress-related and zebrafish studies
}

\author{
Alexandrina Curpan, Ștefan Strungaru, Alexandra Săvucă, Ovidiu Ilie, \\ Alin Ciobîcă, Daniel Timofte, Roxana Cojocariu, \\ Gabriel Plăvan, Mircea Nicoară
}

Alexandrina Curpan - Department of Research, Faculty of Biology, "Alexandru Ioan Cuza" University of Iasi, Carol I Avenue, 20A, Iași, Romania

Ştefan Strungaru - Department of Research, Faculty of Biology, "Alexandru Ioan Cuza" University of Iasi, Carol I Avenue, 20A, Iași, Romania

Alexandra Săvucă - Department of Research, Faculty of Biology, "Alexandru Ioan Cuza" University of Iasi, Carol I Avenue, 20A, Iași, Romania

Ovidiu Ilie - Department of Research, Faculty of Biology, "Alexandru Ioan Cuza" University of Iasi, Carol I Avenue, 20A, Iași, Romania

Alin Ciobîcă - Department of Research, Faculty of Biology, "Alexandru Ioan Cuza” University of Iasi, Carol I Avenue, 20A, Iași, Romania

Daniel Timofte - Faculty of Medicine, "Grigore T. Popa" University of Medicine and Pharmacy, 16th University Street, Iași, Romania

Roxana Cojocariu - Department of Research, Faculty of Biology, "Alexandru Ioan Cuza" University of Iasi, Carol I Avenue, 20A, Iași, Romania

Gabriel Plăvan - Department of Biology, Faculty of Biology, "Alexandru Ioan Cuza” University of Iași, Bd. Carol I, 20A, 700505 Iași, Romania

Mircea Nicoară - Department of Biology, Faculty of Biology, "Alexandru Ioan Cuza" University of Iași, Bd. Carol I, 20A, 700505 Iași, Romania

\section{ABSTRACT}

Modern world is relying more and more on the use of plastics in order to conduct our daily tasks going from the simple lid that covers our morning coffee to large scale use in packaging and buildings. But how does the use of so much plastic influence human's normal growth and development or more importantly, how does it affect the brain functions? For the purpose of this paper of giving a little literature insight over the toxic effects of micro-/nanoplastics we chose to approach it from a multidisciplinary point of view with a focus on the use of zebrafish as animal models; ranging from how microplastics disrupts the host eubiosis and 
\% gradually provokes an impairment along the gut-brain axis (GBA) in both mice and zebrafish to nanoplastics inducing oxidative stress status which furthermore leads to increased mortality and brain cells apoptosis. Nanoplastics has also been reported as being transferred from mother to offsprings causing a series of morphological and physiological changes, as well as neurotransmitters inhibitions (dopamine, oxytocin, serotonin) in zebrafish fish as several studies have suggested.

\section{KEYWORDS:}

Neuropsychiatric disorders, zebrafish, microplastics, gut, oxidative stress.

\section{INTRODUCTION}

Plastics consist of a large variety of polymer types, including polypropylene (PP), polyethylene (PE), polystyrene (PS), polyvinylchloride (PVC), polyethylene terephthalate (PET), polyamides (PA), and so on, which are mainly made from fossil fuels such as petroleum, natural gas, or coal, and are designed to meet the very different needs of end products. It is estimated that at least 8 million tons of plastic waste ends into the world's oceans each year and by 2050 the weight of marine plastics would exceed that of fish. The released plastics are generally subjected to progressive fragmentation under the comprehensive function of environmental physicochemical and biotic factors, such as mechanical abrasion, ultraviolet radiation, and biological degradation by microorganisms.

Micro-/nanoplastics are widespread contaminants. The human body is exposed to microplastics through ingestion of food containing microplastics, inhalation of microplastics in the air and by dermal contact of these particles, contained in products, textiles or in the dust. Ingestion is considered the major route of human exposure to micro/nanoplastics. Due to the evidence of neurotoxicity when testing microplastics in organism or cells and resulting from human exposure to particulate matter, which microplastics are a part of, there is a need to understand how microplastics could be involved in neurotoxicity in humans, contributing to an increased risk of neurodevelopmental disorders (1). These types of disorders represent a challenge for researchers at the worldwide level due to the difficulty of mimicking the whole symptomatology in a laboratory setting. Several neuropsychiatric disorders, such as depression, anxiety and autism $(2,3)$ were linked to the brain-gut impairments accompanied with functional gastrointestinal symptoms. Epilepsy has been induced in zebrafish larvae and adults by exposure to pentylenetetrazol (PTZ) (4), ginkgo toxin (PLP)(5) where it has been shown that there are changes in resting states that lead to hyperactivity and hypersynchronization of the brain (6) by changing GABA levels (5). Moreover, the pattern of co-occurrence of medical comorbidities in autism as compared to any other neurodevelopmental disorders is elevated, especially gastrointestinal disorders (7).

In recent years, a new animal model is gaining more ground in multiple medical and biological fields - zebrafish proving its utility and resemblance to the human nervous system by bringing to the table a series of more favorable features when compared to already established animal models, such as low upkeep cost, fast life cycle, long life (five years) as well as the amount of techniques available to genetically manipulate these vertebrates. Due to the high homology they present with humans (more than $70 \%$ of human genes have orthologues in zebrafish), the relevance of using it at different developmental stages has also improved, with embryos having the advantage of a translucent body and an ability to absorb substances by immersion while exhibiting movements as early as 17 hours post fertilization (muscular contractions) while at 48 hours post fertilization (hpf) they can fully 
respond to stimuli and are able to control their movements $(8,9)$.

Recent statements suggested that more than $99 \%$ of the human faeces samples originated from Asia and Europe contained microplastics. More studies should be conducted to explore the possibility of detecting microplastics in the human body and the effects of microplastics on human health. A reliable methodology for microplastics extraction with application in routine medical analyses is not developed yet. Such studies could raise awareness of the importance of sustainable development and lead to the sustainable development of food and water systems, environmental stewardship, and solutions to this global public health issue. However, there are no current studies focusing on the correlation between micro-/nanoplastics toxicity and neurodevelopmental disorders by the use of zebrafish animal models and human subject making this approach even more interesting.

\section{EFFECTS OF MICRO-/NANOPLASTICS IN HUMAN, MICE AND ZEBRAFISH}

A recent review on the human intake of MPs from food gave an estimate of 39,000-52,000 particles per year depending on age and gender (10). Tap water has been assumed to account for around $10 \%$ of this exposure (4000 particles per year). Consumption of exclusively (mineral) water from bottles would result in an additional exposure of approximately 90,000 MP particles per year. It has been indicated that the estimates should be seen as an underestimation. Combination of exposure from different sources would indicate a current human exposure exceeding 100,000 MP particles per capital during one year, depending on diet and geographic location, and on the particle size range included in the estimate. However, levels in food and water have been considered to be an underestimation. A set of 32 samples of bottled water from Bavaria (Germany) was investigated for the presence of MP particles of the size $1 \mu \mathrm{m}$ and larger (11). The counts of MP particles varied from 2649-2857 (mean \pm standard deviation) liter -1 in single use PET bottles and 4889-5432 liter -1 in water from reusable PET bottles to 6292-10521 liter -1 in glass bottles. Higher counts were found in older reused PET bottles than in new ones, but in all cases the diversity within each group was considerable. Particles smaller than $5 \mu \mathrm{m}$ were identified for approximately $96 \%$ in the PET bottles and $78 \%$ in the glass bottles. In order of abundance, PET (76\%) and PP (10\%) were most commonly found in water from the PET bottles. In glass bottles, PE (46\%), PP (23\%), and a copolymer of styrene and butadiene (14\%) were most common. Two studies have reported on the uptake and bioaccumulation of MPs in mice. In a study by Deng et al. 2017(12), fluorescently labelled PS MPs of 5 and $20 \mu \mathrm{m}$ were daily administered by oral gavage for 1 , $2,4,7,14,21$, and 28 days at a dose of 0.1 $\mathrm{mg}$ per day (corresponding to $1.46 \times 106$ and $2.27 \times 104$ particles 8 of 28 of the 5 and $20 \mu \mathrm{m}$ sizes, respectively). Wash-out groups were exposed for 28 days and sacrificed after a week without treatment. Using light and fluorescence microscopy, MPs were detected in various tissues. Liver, kidney, and gut tissue material was also digested using nitric acid and hydrogen peroxide, and fluorescence in these samples was measured to quantify MP concentrations. These measurements demonstrated gradual accumulation in all tissues that reached a steady state at around 14 days of exposure. After 28 days of exposure, concentrations in the liver, kidney, and gut for the $5 \mu \mathrm{m}$ MPs were reported to be $0.077,0.099$, and $0.417 \mathrm{mg}$ g-1 (ww), respectively, and for the $20 \mu \mathrm{m}$ MPs 0.194, 0.082 , and $0.234 \mathrm{mg} \mathrm{g}-1$ (ww), respectively (levels based on wet weight were provided by Deng and Zhang(12)). After a wash-out period of 1 week, MPs could still be detected in all tissues (levels not reported). An important issue is whether MPs can be absorbed and enter the systemic circulation. Various reports mention that particles $150 \mu \mathrm{m}$ in size or smaller have the potential to cross the intestinal barrier of mammals, whereas particles $20 \mu \mathrm{m}$ in size or smaller may have the potential to penetrate deeply into tissues. Phagocytosis and endocytosis have been named as mechanisms for the uptake of particles smaller than $10 \mu \mathrm{m}$ and persorption in the Peyer's patches of the ileum for 
\%

particles up to $130 \mu \mathrm{m}$ in size has been found, although a low uptake of $0.002 \%$ per $24 \mathrm{~h}$ was reported for the latter process $(13,14)$. When MPs enter tissues, potential effects might include physical stress and damage, apoptosis, necrosis, inflammation, oxidative stress, and immune responses. Diffusion of monomers, toxic substances, or microbes from the particles to the surrounding tissue might contribute to chemical and microbiological hazards. Exposure of zebrafish to MPs [PS; $5 \mu \mathrm{m}$; control, low (50 $\mu \mathrm{g}$ liter-1) and high (500 $\mu \mathrm{g}$ liter-1) exposure] has been found to result in distribution in the gut tissue, as evaluated by histological analysis. Furthermore, inflammatory responses, oxidative stress, and changes in lipid metabolism in the gut tissue have been reported (15).

The antagonistic effect of MPs on the gut microbiota promotes significant long-term repercussions. Not only disrupts the host eubiosis, but also gradually provokes an impairment along the gut-brain axis (GBA) in both mice and zebrafish. Through the analysis of the bacterial hypervariable regions V3-V4 of the 16 rRNA gene, it has been revealed a disruption of the so-called beneficial bacteria in four distinct phyla such as Bacteroidetes, Proteobateria, Firmicutes and Actinobacteria. Subsequent in-depth analysis revealed that twenty-nine intestinal microorganisms identified by operational taxonomic analysis (OUT) changed, of which fifteen at the genus level during exposure to MP. MPs have induced an increased mRNA level of several pro-inflammatory cytokines which explains the metabolic disorders as a result of a dysfunction of the intestinal barrier $(16,17)$.

Continuing this topic and analogous to previous studies, intestinal dysbiosis is associated with fluctuations in mucus volume. A study conducted by Lu et al., 2018(18) has provided a more deepen perspective at the phylum level, MPs being positively correlated with a low richness of Firmicutes and $\alpha$ Proteobacteria following the analysis of the $\mathrm{V} 3-\mathrm{V} 4$ region of the 16 rRNA gene and the overall diversity. Between 310 and 160 gut microbes have been changed after the exposure to MPs, by leading to a decrease of the hepatic triglyceride (TG) and total cholesterol (TCH) level in mice, reflected by a high predisposition towards hepatic lipid disorders.

According to current literature regarding the use of zebrafish embryo and larvae, most studies have been conducted for toxicological assays, but the material and methods are quite similar therefore can be used for studies with a different desired outcome.

With this being said, zebrafish has been used in order to assess the toxic effects of nanoplastics (NPs) on the living organisms, plankton and aquatic life in general. Even if most studies focused on the environmental effects of MPs, over the last years NPs had become a researched topic as "smaller particles are generally more toxic than the corresponding bulk material at the same mass concentration"(19). Studies using $20 \mathrm{~nm}$ diameter polystyrene nanoplastics (PNP) have managed to illustrate the oxidative stress induced by the accumulation of the particles in the brain leading to increased mortality and brain cells apoptosis, illustrating as well their ability to cross the blood-brain barrier - with oxidative stress being one of the possible underlying theories of neurodevelopmental disorders. When applied to other animal models, it was suggested that PNP can affect the nervous system with focus on the behavioral changes, like reduced motor activity and severe seizure-like behavior (20, 21). Several studies have demonstrated that NPs can be transmitted from mother to offspring causing morphological and physiological alterations such as increased mortality in embryo and increased yolk area, spine curvature, caudal flexure etc. in larvae as well as decreased cyclooxygenase (COX) activity in embryo and inhibition of a series of neurotransmitters (dopamine - thought to be also involved in schizophrenia, oxytocin, serotonin, melatonin, low acetylcholinesterase activity). In larvae, NPs can accumulate in mouth, gut, blood, liver, heart, gills, muscles and brain, but the interaction of NPs in the intestinal tract affects the gut microbiome and leads to inflammation (gastrointestinal system of zebrafish is homologous to the mammalian 


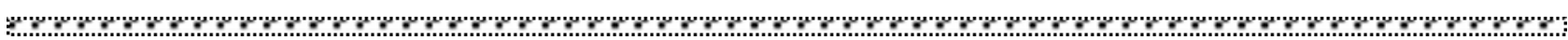

one), concomitantly with a disruption of the gut metabolism through an abnormal gene expression and energy production (22).

Studies using zebrafish have opened up new perspectives as it turned out that recurrent light-sensitive effects (23) and some parameters increased with exposures to PTZ concentrations that led to the cumulative frequency of the score, the intensity of seizures and their severity, latency to onset, mortality rate $(6,24)$ all opening avenues of research for new genetic markers of epilepsy in zebrafish (25).

As previously stated, zebrafish are capable of exhibiting complex behavior as early as 48 hours post fertilization corresponding to the larvae stage. Similar to other animal models, the behavior of this teleost can be assessed with a plethora of tests such as the light-dark test, novel tank test for anxiety with the unique behavior of freezing and diving to the bottom, T-maze, plus maze and social preference, as well as the acoustic startle reflex that is used to test the ability to learn as well as memory based on the habituation ability represented in this case by a rapid escape response - C-start, which is sensible to $\mathrm{N}$-methyl-D-aspartate receptor (NMDAR) blockade (neurotransmitter involved in schizophrenia's etiology). Another test that can be conducted on zebrafish larvae that is also used to test the short- and long-term memory of zebrafish is visual lateralization (novel object recognition test) with zebrafish having a preference of using left eye system for appraising novelty. The last two tests being specific for this animal model.

Whether it is about the way of communication through the vagus nerve (26), the immune(27) or neuroendocrine pathways (28), or even microorganism-derived neuroactive chemicals (29), the undoubted connection between gut and brain has been the subject of countless studies.

It has been shown that the bidirectional braingut axis disturbance could be involved in the abnormal function of the enteric or/and central nervous systems (CNS), this being an additional argument that links CNS to mechanisms that may have a role in the pathogenesis of functional gastrointestinal disorders (30). Regarding one of them, irritable bowel syndrome (IBS), it has been shown that stress plays an essential role in the occurrence of its symptoms, given the fact that it can modulate the hypothalamicpituitary-adrenal (HPA) axis activity $(31,32)$. Also, inflammatory bowel disease (IBD), which is a chronic relapsing disorder with a progressive and destructive nature (33), is linked to risk factors such as microbial dysbiosis, or exposure to toxins including xenoestrogens. In this regard, recent findings indicated that bisphenol-A (BPA), which is usually found in polycarbonate plastics and epoxy resins, is correlated with colonic inflammation. Moreover, BPA not only has the ability to exacerbate colonic inflammation, but alter microbiota metabolites derived from aromatic amino acids in an acute dextran sulfate sodiuminduced colitis animal model, increasing mortality and aggravating disease activity as well as inflammation in the middle colon (34).

Even if the exact communication pathways mechanism between the brain and gut are still unclear, it seems that the gut microbiome influences brain and behavior, using microbiota-gut-brain axis $(35,36)$, in this way, understanding why the microbiome influences behavior requires a focus on microbial ecology and local effects within the host. The exact underlying mechanism of how the homeostasis of this microscopic environment is gradually disrupted in humans is obscure. Unfortunately, in the current literature no study that addresses such a perspective exists, everything remaining at the stage of hypothesis. However, one pilot study has provided conclusive evidence in this context, and indeed demonstrated the existence of MPs in the human stool, which could constitute the missing link (37).

In fact, our group is currently working on further understanding and establishing the potential of zebrafish embryos and larvae in the study of neurodevelopmental disorders, as 
r.m\%\%

well as studying the current interactions between nanoplastics, microbiota changes and neurodevelopmental disorders and their importance, understanding the connection between micro-/nanoplastics pollution and physiological and behavioral changes and also establishing oxidative stress status disruption that was observed in schizophrenia, autism and MPs/NPs toxicity assays in order to assess its implications in the etiology of these disorders.

\section{CONCLUSION}

The need to reduce our plastic usage becomes more critical as days are passing by and more studies are bringing to light the harmful effects of plastics at a micro and nano level. Studies using zebrafish have opened up new perspectives due to their anatomical similarities to human and rodents, especially the existence of the blood-brain barrier.

Even if a direct, clean-cut connection can't be established between micro-/nanoplastics pollution and neuropsychiatric disorders, a more indirect one could be observed by the effects of plastics on gut microbiota as brain-gut impairments has been previously linked to several neuropsychiatric disorders such as depression, anxiety and autism, even by our team. The hypothesis that oxidative stress might be an underlying mechanism of psychiatric disorders such as schizophrenia and autism and the observation that plastics can induce a disruptance of the oxidative status requires further investigations and studies on whether plastics induce neuropsychiatric disorders by impairments at the oxidative status and gut level or not, and we recommend the use of zebrafish for this purpose.

\section{ACKNOWLEDGEMENTS AND DISCLOSURES}

Ciobîcă A. is currently supported by a UEFISCDI Research Grant for Young Research Teams Support, number PN-III-P1-1.1-TE2016-1210, named "Complex study on oxidative stress status, inflammatory processes and neurological manifestations correlations in irritable bowel syndrome pathophysiology (animal models and human patients)." Also, Cojocariu RO was funded by the European Social Fund, through Operational Programme Human Capital 2014-2020, project number POCU/380/6/13/123623, project title "PhD Students and Postdoctoral Researchers Prepared for the Labour Market!"

\section{REFERENCES}

1. Prata JC, da Costa JP, Lopes I et al. Environmental exposure to microplastics: An overview on possible human health effects. Sci Total Environ. 2020.1;702. Available from: https://pubmed.ncbi.nlm.nih.gov/31733547/

2. O'malley D, Quigley EMM, Dinan TG, Cryan JF. Do interactions between stress and immune responses lead to symptom exacerbations in irritable bowel syndrome? Brain Behav Immun. 2011;25(7):1333-41. https://pubmed.ncbi.nlm.nih.gov/21536124/

3. Singh P, Agnihotri A, Pathak MK et al. Psychiatric, somatic and other functional gastrointestinal disorders in patients with irritable bowel syndrome at a tertiary care center. J Neurogastroenterol Motil. 2012;18(3):324-31. https://pubmed.ncbi.nlm.nih.gov/22837881/

4. Burrows DRW, Samarut, Liu J et al. Imaging epilepsy in larval zebrafish. Eur J Paediatr Neurol. 2020;24:70-80. https://doi.org/10.1016/j.ejpn.2020.01.006

5. Lee GH, Sung SY, Chang WN et al. Zebrafish larvae exposed to ginkgotoxin exhibit seizure like behavior that is relieved by pyridoxal-5'-phosphate, GABA and anti-epileptic drugs. DMM Dis Model Mech. 2012;5(6):785-95.

6. Diaz Verdugo C, Myren-Svelstad S, Aydin E et al. Glia-neuron interactions underlie state transitions to generalized seizures. Nat Commun. 2019;10(1):1-13.

7. Pulikkan J, Mazumder A, Grace T. Role of the Gut Microbiome in Autism Spectrum Disorders. Adv Exp Med Biol. 2019;1118:253-69. 
2. 8. Spence R, Gerlach G, Lawrence C, Smith C. The behaviour and ecology of the zebrafish, Danio rerio. Biol Rev. 2008;83(1):13-34.

9. Vaz R, Hofmeister W, Lindstrand A. Zebrafish Models of Neurodevelopmental Disorders: Limitations and Benefits of Current Tools and Techniques. Int J Mol Sci. 2019;20(6):1-26.

10. Cox KD, Covernton GA, Davies HL et al. Human Consumption of Microplastics. Environ Sci Technol. 2019;53(12):7068-74.

11. Oßmann BE, Sarau G, Holtmannspötter H et al. Small-sized microplastics and pigmented particles in bottled mineral water. Water Res. 2018;141:307-16.

12. Deng Y, Zhang Y, Lemos B, Ren H. Tissue accumulation of microplastics in mice and biomarker responses suggest widespread health risks of exposure. Sci Rep. 2017;7(1):1-10.

13. Revel M, Châtel A, Mouneyrac C. Micro(nano)plastics: A threat to human health? Curr Opin Environ Sci Heal. 2018;1:17-23.

14. Academies SA for P by E. A Scientific Perspective on Microplastics in Nature and Society. Berlin; 2019. https://www.sapea.info/wp-content/uploads/report.pdf

15. Qiao R, Sheng C, Lu Y et al. Microplastics induce intestinal inflammation, oxidative stress, and disorders of metabolome and microbiome in zebrafish. Sci Total Environ. 2019;662:246-53.

16. Jin Y, Xia J, Pan Z et al. Polystyrene microplastics induce microbiota dysbiosis and inflammation in the gut of adult zebrafish. Environ Pollut. 2018;235:322-9.

17. Jin Y, Lu L, Tu W et al. Impacts of polystyrene microplastic on the gut barrier, microbiota and metabolism of mice. Sci Total Environ. 2019;649:308-17.

18. Lu L, Wan Z, Luo T et al. Polystyrene microplastics induce gut microbiota dysbiosis and hepatic lipid metabolism disorder in mice. Sci Total Environ. 2018;631-632:449-58.

19. Mattsson K, Johnson E V., Malmendal A et al. Brain damage and behavioural disorders in fish induced by plastic nanoparticles delivered through the food chain. Sci Rep. 2017;7(1):1-7.

20. Sökmen TÖ, Sulukan E, Türkoğlu M et al. Polystyrene nanoplastics $(20 \mathrm{~nm})$ are able to bioaccumulate and cause oxidative DNA damages in the brain tissue of zebrafish embryo (Danio rerio). Neurotoxicology. 2020;77:51-9.

21. Bhagat J, Zang L, Nishimura N, Shimada Y. Zebrafish: An emerging model to study microplastic and nanoplastic toxicity. Vol. 728, Science of the Total Environment. Elsevier B.V.; 2020. p. 138707.

22. Wan Z, Wang C, Zhou J et al. Effects of polystyrene microplastics on the composition of the microbiome and metabolism in larval zebrafish. Chemosphere. 2019;217:646-58.

23. Sun S, Zhu C, Ma M et al. A pentylenetetrazole-induced kindling zebrafish larval model for evoked recurrent seizures. bioRxiv. 2019;787580.

24. Mussulini BHM, Leite CE, Zenki $\mathrm{KC}$ et al. Seizures Induced by Pentylenetetrazole in the Adult Zebrafish: A Detailed Behavioral Characterization. Callaerts P, editor. PLoS One. 2013;8(1):e54515.

25. Stewart AM, Desmond D, Kyzar E et al. Perspectives of zebrafish models of epilepsy: What, how and where next? Brain Res Bull. 2012;87(2-3):135-43.

26. Forsythe P, Bienenstock J, Kunze WA. Vagal Pathways for Microbiome-Brain-Gut Axis Communication BT Microbial Endocrinology: The Microbiota-Gut-Brain Axis in Health and Disease. In: Lyte M, Cryan JF, editors. New York, NY: Springer New York; 2014.115-33.

27. Fung TC, Olson CA, Hsiao EY. Interactions between the microbiota, immune and nervous systems in health and disease. Nat Neurosci. 2017;20(2):145-55.

28. Neuman H, Debelius JW, Knight R, Koren O. Microbial endocrinology: the interplay between the microbiota and the endocrine system. FEMS Microbiol Rev. 2015;39(4):509-21.

29. Lyte M. Microbial endocrinology in the microbiome-gut-brain axis: how bacterial production and utilization of neurochemicals influence behavior. PLoS Pathog. 2013/11/14. 2013;9(11):e1003726-e1003726.

30. Karantanos T, Markoutsaki T, Gazouli M et al. Current insights in to the pathophysiology of Irritable Bowel Syndrome. Gut Pathog. 2010;2(1):3.

31. Musial F, Häuser W, Langhorst J. Psychophysiology of visceral pain in IBS and health. J Psychosom Res. 2008;64(6):589-97.

32. Qin HY, Cheng CW, Tang XD, Bian ZX. Impact of psychological stress on irritable bowel syndrome. World J Gastroenterol. 2014;20(39):14126-31.

33. Neurath MF. Current and emerging therapeutic targets for IBD. Nat Rev Gastroenterol Hepatol. 2017;14(5):26978. 

34. DeLuca JAA, Allred KF, Menon R et al. Bisphenol-A alters microbiota metabolites derived from aromatic amino acids and worsens disease activity during colitis. Exp Biol Med. 2018;243(10):864-75.

35. Crumeyrolle-Arias M, Jaglin M, Bruneau A et al. Absence of the gut microbiota enhances anxiety-like behavior and neuroendocrine response to acute stress in rats. Psychoneuroendocrinology.2014;42:207-17.

36. Foster JA, Rinaman L, Cryan JF. Stress \& the gut-brain axis: Regulation by the microbiome. Neurobiol Stress. 2017;7:124-36.

37. Liebmann B, Köppel S, Königshofer P et al. Assessment of microplastic concentrations in human stool Preliminary results of a prospective study. 2018.

\section{Correspondence}

Daniel Timofte,

$\mathrm{MD}, \mathrm{PhD}$, professor, Faculty of Medicine, "Grigore T. Popa” University of Medicine and Pharmacy, 16th University Street, Iasi, Romania, dantimofte@ yahoo.com

Submission: 20 jul. 2020

Acceptance: 02 sep. 2020 